\title{
Gender, HIV-Related Stigma, and Health-Related Quality of Life Among Adults Enrolling in HIV Care in Tanzania
}

\author{
Angela M. Parcesepe ${ }^{1,2} \cdot$ Denis Nash $^{2,3} \cdot$ Olga Tymejczyk $^{2,3} \cdot$ William Reidy $^{4} \cdot$ Sarah Gorrell Kulkarni $^{2} \cdot$ Batya Elul $^{4}$
}

Published online: 30 March 2019

๑) Springer Science+Business Media, LLC, part of Springer Nature 2019

\begin{abstract}
HIV-related stigma has been associated with worse health-related quality of life (HRQoL) among people living with HIV (PLWH). Little is known about how different types of HIV-related stigma (i.e., anticipatory, internalized, or enacted HIVrelated stigma) influence HRQoL and whether these relationships differ by gender. The sample included 912 PLWH aged 18 years or older enrolling in HIV care at four health facilities in Tanzania. HRQoL was assessed with the life satisfaction and overall function subscales of the HIV/AIDS-Targeted Quality of Life (HAT-QoL) instrument. Sex-stratified multivariable logistic regression modeled the association of anticipatory, internalized, and enacted HIV-related stigma on poor HRQoL. Across all participants, the mean life satisfaction score was 63.4 (IQR: 43.8, 81.3) and the mean overall function score was 72.0 (IQR: 58.3, 91.7). Mean HRQoL scores were significantly higher for women compared to men for overall function (5.1 points higher) and life satisfaction (4.3 points higher). Fourteen percent of respondents reported recent enacted HIV-related stigma and 13\% reported recent medium or high levels of internalized stigma. In multivariable models, high internalized and high anticipatory stigma were significantly associated with higher odds of poor life satisfaction and poor overall function in both men and women. Psychosocial interventions to prevent or reduce the impact of internalized and anticipatory stigma may improve HRQoL among persons in HIV care. Future research should longitudinally examine mechanisms between HIV-related stigma, poor HRQoL, and HIV care outcomes.
\end{abstract}

Keywords HIV $\cdot$ Stigma $\cdot$ Quality of life $\cdot$ Tanzania

Angela M. Parcesepe

angela_parcesepe@unc.edu

Denis Nash

denis.nash@sph.cuny.edu

Olga Tymejczyk

olga.tymejczyk@sph.cuny.edu

William Reidy

wr2205@columbia.edu

Sarah Gorrell Kulkarni

sarah.kulkarni@sph.cuny.edu

Batya Elul

be2124@columbia.edu

1 Department of Maternal and Child Health, Gillings School of Global Public Health, University of North Carolina at Chapel Hill, Chapel Hill, NC, USA

2 Institute for Implementation Science in Population Health, City University of New York, New York, NY, USA

3 Graduate School of Public Health and Health Policy, City University of New York, New York, NY, USA

4 Department of Epidemiology, Columbia University, New York, NY, USA

\section{Introduction}

The World Health Organization recommends antiretroviral therapy (ART) for all people living with HIV (PLWH), regardless of CD4 cell count [1]. This policy, often referred to as Treat All, is informed by evidence that earlier HIV treatment is associated with improved outcomes across the HIV care continuum and reduced HIV transmission [2-5]. In recent years, as access to ART has expanded, due in part to the implementation and scale-up of Treat All policies, HIV-related morbidity and mortality have decreased and life expectancy among PLWH has increased [6, 7]. In many settings, treatment of HIV has shifted to a chronic care model of disease management [8]. Given these shifts in the treatment and clinical outcomes among PLWH, greater attention is needed to their health-related quality of life (HRQoL) and potentially modifiable factors that influence HRQoL among PLWH.

Though precise definitions vary, HRQoL is a multidimensional construct that reflects one's quality of life through 
assessment of physical, mental, emotional, and social functioning [9]. Poor HRQoL has been associated with suboptimal HIV treatment outcomes including worse engagement in care, poor ART adherence, and increased mortality, as well as poor mental health in both resource-rich and resourcepoor settings [10-13]. Greater understanding of factors that influence HRQoL among PLWH is critical to identifying effective strategies to improve the health and well-being of PLWH.

HIV-related stigma is common among PLWH and associated with poor HRQoL [14-16]. HIV-related stigma can take many forms including anticipatory stigma, internalized stigma, and enacted stigma. Anticipatory stigma concerns individuals' expectations of experiencing enacted HIVrelated stigma as a result of being HIV-positive. Enacted HIV-related stigma involves experiencing stigmatizing behaviors or negative treatment due to one's HIV status. Internalized HIV-related stigma occurs when an individual adopts stigmatizing beliefs about PLWH and applies these stigmatizing beliefs to themselves. In the framework of the relationship between HIV-related stigma and the health and well-being of PLWH [17], Earnshaw and Chaudoir posit that different dimensions of HIV-related stigma may differentially impact health-related outcomes, including HRQoL, and emphasize the importance of understanding the relationship between HIV-related stigma and quality of life disaggregated by type of stigma and type of well-being measure assessed [17]. Despite consistent findings that HIV-related stigma is associated with worse HRQoL, understanding of how different dimensions of stigma (e.g., internalized, anticipated, or enacted) are related to different components of HRQoL, remains limited, particularly in sub-Saharan Africa, which continues to bear a disproportionate burden of the HIV epidemic. A more nuanced understanding of the relationship between HIV-related stigma and HRQoL is warranted.

Previous research has found differences in how men and women living with HIV perceive and experience HIVrelated stigma [13, 18, 19]. For example, among PLWH in the United States, HIV disclosure concerns were associated with increased health-related worries among women, but not among men living with HIV [20]. Among PLWH in China, women living with HIV endorsed significantly higher internalized HIV-related stigma compared to men [21]. Gendered experiences of HIV-related stigma may be informed by differences in the social and economic position of men and women across societies and by the social construction of both stigma and gender across settings. Previous research has found that women living with HIV often indicate concerns about the real or anticipated social and relational impact of HIV-related stigma in their lives and the lives of their children or other family members, including social rejection, isolation, and violence [22]. Research with women living with HIV in the U.S. found that women's decision-making around HIV disclosure prioritized protecting social relationships and minimizing potential negative social effects of disclosure [22]. Given these gendered differences in experiences of HIV-related stigma, the relationship between HIV-related stigma and HRQoL may be meaningfully different for men and women living with HIV. However, little is known regarding whether the relationship between types of HIV-related stigma and types of HRQoL differs by gender. It is important to understand to what extent HIV-related stigma and HRQoL function differently among men and women. The objectives of this analysis were (1) to examine the relationship between three dimensions of HIV-related stigma (anticipatory, internalized, and enacted stigma) and two components of HRQoL (overall function and life satisfaction) and (2) to assess whether these relationships differ by gender.

\section{Methods}

Data were collected as part of a study conducted at four HIV treatment clinics in the Kagera region of northwestern Tanzania. All clinics were supported by the Tanzanian Ministry of Health with technical assistance from ICAP at Columbia University via funding from the President's Emergency Plan for AIDS Relief (PEPFAR) at the time of data collection. Individuals were eligible to participate in the study if they were: (1) aged 18 years and older, (2) newly enrolling in HIV care and (3) not known to be eligible for ART (based on World Health Organization (WHO) stage and/or CD4 count) at enrollment in HIV care between March 2012-November 2013. From March 2012 until April 2013, the Tanzanian national guidelines recommended ART initiation for patients with WHO stage IV or CD4 + count $<200$ cells/ $\mu \mathrm{L}$, or WHO III with CD $4+<350$ cells $/ \mu \mathrm{L}$; in April 2013 the guidelines were expanded to include all patients with CD $4+$ count $<350$ cells $/ \mu \mathrm{L}$. The original study enrolled 920 individuals. The current sample is comprised of 912 individuals for whom data on HRQoL and gender were available. The study was approved by the Institutional Review Boards of the National Institute for Medical Research of the United Republic of Tanzania, Columbia University Medical Center, and the City University of New York.

\section{Data Collection}

Patients potentially eligible for study participation were identified by providers within 90 days of their first clinic visit. Interested patients were referred to study staff for eligibility screening and consent procedures. Data collection consisted of a structured interview conducted by trained research assistants which included questions on 
sociodemographics, HIV-related stigma, and health-related quality of life.

\section{Measures}

\section{Health-Related Quality of Life}

Health-related quality of life was assessed using the HIV/ AIDS-Targeted Quality of Life (HAT-QoL) instrument. The HAT-QoL has demonstrated reliability and validity among PLWH $[23,24]$ in resource-poor settings, including sub-Saharan Africa [25, 26]. This analysis includes two subscales of the HAT-QoL: the overall function subscale (6 items) and the life satisfaction subscale (4 items). Participants were asked to report on how often they were able to complete a given health-related task or felt a particular way in the previous 4 weeks. Response options ranged from 1 (none of the time) to 5 (all of the time). Scores for each subscale were transformed linearly to a possible range of $0-100$, according to a prescribed algorithm, with higher values indicating better functioning and higher life satisfaction.

\section{Recent Internalized Stigma}

Internalized stigma in the past 3 months was measured with the 5-item negative self-perception subscale (e.g. You felt that you did not deserve to live, You felt that you were no longer a person) of the HIV/AIDS Stigma Instrument, PLWHA (HASI-P), developed in sub-Saharan Africa [27]. An additional item not from this scale (You thought someone had cursed you) also was included. Response options ranged from Never (4) to Most of the time (1) and were recoded so that higher scores represented higher internalized stigma (Cronbach's alpha $=0.86)$. Responses were categorized into three categories: none, low, and medium or high levels of internalized stigma.

\section{Recent Anticipatory Stigma}

Study investigators knew of no scales validated in sub-Saharan Africa to measure anticipatory HIV-related stigma at the time of data collection. Thus, anticipatory stigma was assessed with 12 Yes/No items created in accordance with the concept described by Earnshaw and Chaudoir (e.g., If others know or suspect you are HIV positive, your partner might get violent; your children might be abused or discriminated against; family members might treat you differently). A total anticipatory stigma score was constructed as the proportion of endorsed items from among all questions the participant was eligible to answer (i.e., participants without children or without a partner were not eligible to answer those respective items) (Cronbach's alpha $=0.65$ ).
Anticipatory stigma scores were categorized into tertiles based on distribution in the study sample.

\section{Recent Enacted Stigma}

Enacted stigma in the past 3 months was measured with eight items selected from HIV/AIDS Stigma InstrumentPLWA (HASI-P) subscales (verbal abuse, fear of contagion, and social isolation); items inquired about how often the participant had experienced rejection due to their HIV status (e.g., You were told that you have no future, You were told that God is punishing you, Someone stopped being your friend), with response options ranging from Never (4) to Most of the time (1) (Cronbach's alpha =0.70) [27]. Because the reported frequency of occurrence of all items was low, the summary measure was coded as any recent enacted HIVrelated stigma versus no recent enacted HIV-related stigma.

\section{Sociodemographic Characteristics}

Sociodemographic variables included age, sex, education, religion, relationship status, employment status, food insufficiency, and time away from home.

\section{Statistical Analysis}

Univariate analyses were conducted to assess the mean overall function and life satisfaction scores for the entire sample population and separately by gender. Sex-stratified analyses of differences in group mean HRQoL scores by type of HIV-related stigma were conducted using t-tests or ANOVA procedures, as appropriate. Logistic regression was used to model the association of HIV-related stigma and poor HRQoL separately by gender. In multivariable analyses, poor quality of life was defined as being in the lowest quality of life quartile as compared to the top three quartiles. Adjusted analyses controlled for age, relationship status, and employment status. All analyses accounted for clustering by health facility using proc survey logistic in SAS Version 9.4. Because internalized stigma was highly correlated with enacted stigma, multivariable regression models were run separately to examine the relationship between each type of HIV-related stigma and HRQoL.

\section{Results}

Of the 912 participants included in this analysis, $63.2 \%$ were female (Table 1). The median age was 35 years (Interquartile range [IRQ]: 28,42$)$. Three quarters $(77.9 \%)$ of participants had ever attended school. The majority of participants $(58.1 \%)$ were married or in a relationship. Most participants (61.8\%) were Catholic. Past-year food insufficiency was 
Table 1 Sociodemographic characteristics and HIV-related stigma by health-related quality of life among adults enrolling in HIV care and not known to be eligible for ART in Tanzania

\begin{tabular}{|c|c|c|c|c|c|}
\hline \multirow[t]{2}{*}{ Sociodemographic characteristics } & \multirow[t]{2}{*}{ Overall n (\%) } & \multicolumn{2}{|c|}{ Overall function } & \multicolumn{2}{|c|}{ Life satisfaction } \\
\hline & & Mean & $p$ value & Mean & $p$ value \\
\hline \multicolumn{6}{|l|}{ Sex } \\
\hline Male & $336(36.8)$ & 68.8 & 0.0038 & 60.7 & 0.0161 \\
\hline Female & $576(63.2)$ & 73.9 & & 65.0 & \\
\hline \multicolumn{6}{|l|}{ Ever attended school } \\
\hline Yes & $710(77.9)$ & 72.4 & 0.3727 & 63.9 & 0.2115 \\
\hline No & $202(22.1)$ & 70.6 & & 61.4 & \\
\hline \multicolumn{6}{|l|}{ Relationship status $^{\mathrm{a}}$} \\
\hline Married or in a relationship & $529(58.1)$ & 75.4 & $<0.0001$ & 65.8 & $<0.0001$ \\
\hline Never married & $42(4.6)$ & 64.9 & & 50.3 & \\
\hline Widowed & $118(13.0)$ & 70.3 & & 64.3 & \\
\hline Divorced/separated & $222(24.4)$ & 66.0 & & 59.4 & \\
\hline Age $^{\mathrm{a}}$ & & & 0.0241 & & 0.5297 \\
\hline $18-25$ & $120(13.2)$ & 76.0 & & 61.4 & \\
\hline $26-40$ & $532(58.7)$ & 72.8 & & 64.0 & \\
\hline $41-50$ & $172(19.0)$ & 68.4 & & 63.1 & \\
\hline$>50$ & $82(9.1)$ & 68.0 & & 60.2 & \\
\hline Religion & & & 0.2342 & & 0.8624 \\
\hline Catholic & $564(61.8)$ & 72.5 & & 63.4 & \\
\hline Protestant & $205(22.5)$ & 73.0 & & 64.4 & \\
\hline Muslim & $72(7.9)$ & 71.5 & & 63.1 & \\
\hline Seventh day adventist & $10(1.1)$ & 57.9 & & 58.1 & \\
\hline Born again & $58(6.4)$ & 66.4 & & 60.5 & \\
\hline Other/none & $3(0.3)$ & 73.6 & & 54.2 & \\
\hline \multicolumn{6}{|c|}{ Frequency of food insufficiency in past year ${ }^{a}$} \\
\hline Never/seldom & $766(84.9)$ & 73.6 & $<0.0001$ & 65.9 & $<0.0001$ \\
\hline Sometimes/often/always & $136(15.1)$ & 63.2 & & 50.0 & \\
\hline \multicolumn{6}{|l|}{ Currently employed for payment } \\
\hline No & $352(38.9)$ & 72.0 & 0.9446 & 61.6 & 0.0133 \\
\hline Yes & $553(61.1)$ & 71.9 & & 66.0 & \\
\hline \multicolumn{6}{|c|}{ Away from home $>1$ month in last year ${ }^{a}$} \\
\hline Yes & $253(27.9)$ & 69.1 & 0.0309 & 62.0 & 0.3213 \\
\hline No & $655(72.1)$ & 73.0 & & 63.9 & \\
\hline \multicolumn{6}{|l|}{ Study site } \\
\hline Mugana & $168(18.4)$ & 79.6 & $<0.0001$ & 80.7 & $<0.0001$ \\
\hline Ndolage & $269(29.5)$ & 72.1 & & 59.8 & \\
\hline Nyakahanga & $268(29.4)$ & 67.5 & & 52.2 & \\
\hline Rubya & $207(22.7)$ & 71.5 & & 68.4 & \\
\hline \multicolumn{6}{|l|}{ HIV-related stigma } \\
\hline \multicolumn{6}{|l|}{ Enacted stigma $^{a}$} \\
\hline None & $716(86.0)$ & 73.6 & 0.0151 & 66.4 & $<0.0001$ \\
\hline Any & $117(14.0)$ & 67.6 & & 56.6 & \\
\hline \multicolumn{6}{|l|}{ Internalized stigma ${ }^{a}$} \\
\hline None & $487(53.8)$ & 73.9 & $<0.0001$ & 65.9 & $<0.0001$ \\
\hline Low & $304(33.6)$ & 73.1 & & 65.2 & \\
\hline \multirow[t]{2}{*}{ Medium or high } & $115(12.7)$ & 61.9 & & 48.9 & \\
\hline & Mean (range) & & & & \\
\hline Anticipatory stigma ${ }^{a}$ & $23.0(0-100)$ & & & & \\
\hline Bottom tertile (least stigma) & $2.9(0-10)$ & 78.8 & $<0.0001$ & 70.7 & $<0.0001$ \\
\hline Middle tertile & $18.7(11-27)$ & 70.5 & & 63.5 & \\
\hline Top tertile (most stigma) & $49.7(28-100)$ & 66.3 & & 55.8 & \\
\hline
\end{tabular}

a Missing by variable: relationship status $n=1$, age $n=6$, food insufficiency $n=10$, away from home for $>1$ month $n=4$, enacted stigma $n=79$, internalized stigma $n=6$, anticipatory stigma $n=7$ 
relatively uncommon with $84.9 \%$ reporting never or seldom experiencing food insufficiency in the past year. The majority of participants were currently working for pay $(61.1 \%)$ and had not spent more than 1 month away from home in the past year (72.1\%). Most participants (78.1\%) had disclosed their HIV status to someone. Among those who disclosed their status to someone $(n=712), 51.8 \%$ had disclosed their status to a spouse or partner, $33.3 \%$ had disclosed their status to a parent, $21.8 \%$ had disclosed their status to a friend, and $13.5 \%$ had disclosed their status to a child.

Across all participants, the mean life satisfaction score was 63.4 (IQR: 43.8, 81.3) and the mean overall function score was 72.0 (IQR: 58.3, 91.7). In bivariate analyses, mean HRQoL scores were significantly higher for women compared to men for overall function (5.1 points higher) and life satisfaction (4.3 points higher) (Fig. 1). As shown in Table 1, for both overall function and life satisfaction, mean scores were highest among participants who were married or in a

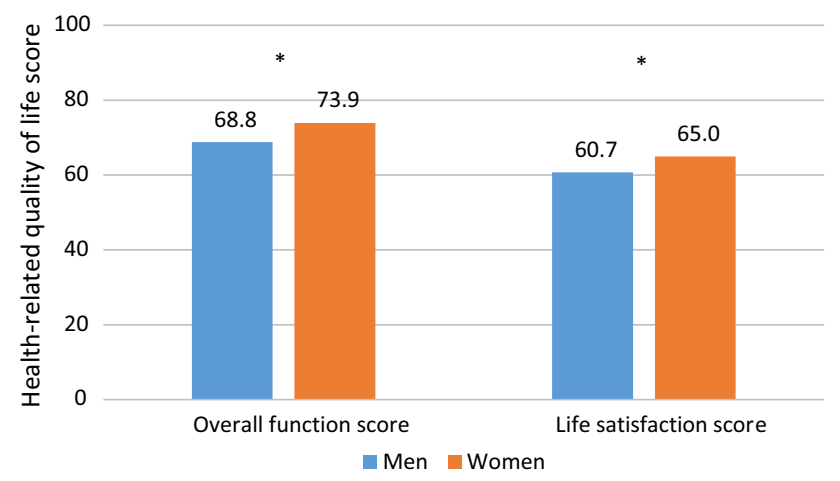

Fig. 1 Mean health-related quality of life scores by gender among adults enrolling in HIV care and not known to be eligible for ART in Tanzania relationship and lowest among participants who had never been married. Similarly, food insufficiency was significantly associated with mean overall function and life satisfaction scores, with those reporting experiencing food insufficiency never or seldom in the past year reporting significantly higher mean overall function $(p<0.0001)$ and life satisfactions $(p<0.0001)$ scores compared to those who reported food insufficiency sometimes, often, or always in the past year. Employment status was significantly associated with mean life satisfaction score $(p=0.0133)$, but not with mean overall function score, while having been away from home for more than 1 month in the past year was significantly associated with mean overall function score $(p=0.0309)$, but not with mean life satisfaction score.

Fourteen percent of respondents reported having experienced recent enacted HIV-related stigma and $12.7 \%$ reported recent medium or high levels of internalized stigma. In relation to anticipatory stigma, almost two-thirds (63.5\%) of respondents indicated concern that people might gossip about them if they knew or suspected that they were HIVpositive. Almost half $(46.4 \%)$ of respondents with children worried that their children might become upset or fearful if they knew or suspected that the respondent was HIVpositive. In bivariate analyses, experience of each form of stigma (anticipatory, internalized, and enacted HIV-related stigma) was associated with lower mean HRQoL scores (Table 2). For both anticipated and internalized stigma, higher levels of stigma were associated with significantly lower mean overall function and life satisfaction scores overall and when stratified by gender. Mean HRQoL scores were significantly higher for those who reported no internalized stigma compared to those who reported medium or high levels of internalized stigma for overall function (women: 12.3 points higher, $p<0.0001$; men: 12.6 points higher, $p=0.04$ )

Table 2 HIV-related stigma and health-related quality of life among adults enrolling in HIV care and not known to be eligible for ART in Tanzania by gender

\begin{tabular}{|c|c|c|c|c|c|c|c|c|c|c|c|c|}
\hline & \multicolumn{6}{|c|}{ Mean overall function score } & \multicolumn{6}{|c|}{ Mean life satisfaction score } \\
\hline & \multicolumn{3}{|c|}{ Women } & \multicolumn{3}{|c|}{ Men } & \multicolumn{3}{|c|}{ Women } & \multicolumn{3}{|l|}{ Men } \\
\hline & $\mathrm{n}$ & Mean & $p$ value & $\mathrm{n}$ & Mean & $p$ value & $\mathrm{n}$ & Mean & $p$ value & $\mathrm{n}$ & Mean & $p$ value \\
\hline \multicolumn{13}{|l|}{ Enacted stigma } \\
\hline None & 438 & 75.7 & 0.006 & 278 & 70.4 & 0.48 & 438 & 67.9 & 0.005 & 278 & 64.1 & 0.0009 \\
\hline Any & 84 & 67.9 & & 33 & 66.9 & & 84 & 59.4 & & 33 & 49.1 & \\
\hline \multicolumn{13}{|l|}{ Internalized stigma } \\
\hline None & 309 & 75.7 & $<0.0001$ & 178 & 70.7 & 0.04 & 309 & 67.5 & $<0.0001$ & 178 & 63.1 & 0.0001 \\
\hline Low & 184 & 75.6 & & 120 & 69.1 & & 184 & 66.8 & & 120 & 62.5 & \\
\hline Medium or high & 82 & 63.4 & & 33 & 58.1 & & 82 & 51.3 & & 33 & 43.2 & \\
\hline \multicolumn{13}{|l|}{ Anticipatory stigma } \\
\hline Bottom tertile (least stigma) & 177 & 80.9 & $<0.0001$ & 128 & 76.0 & 0.0003 & 177 & 72.7 & $<0.0001$ & 128 & 68.2 & $<0.0001$ \\
\hline Middle tertile & 206 & 73.7 & & 112 & 64.8 & & 206 & 65.9 & & 112 & 59.2 & \\
\hline Top tertile (most stigma) & 188 & 67.7 & & 94 & 63.4 & & 188 & 57.5 & & 94 & 52.3 & \\
\hline
\end{tabular}


and life satisfaction (women: 16.2 points higher, $p<0.0001$; men: 19.9 points higher, $p<0.0001$ ). A similar pattern was observed between anticipatory stigma and HRQoL for both women and men. In bivariate analyses, mean life satisfaction scores were significantly higher among those who reported no enacted stigma compared to those who reported having experienced enacted stigma overall ( 9.8 points higher) and separately among women and men (women: 8.5 points higher, $p=0.005$; men: 15.0 points higher, $p=0.0009$ ). Having experienced enacted stigma was associated with significantly lower overall function score among women, but not among men ( 7.8 points lower among women vs 3.5 points lower among men).

In adjusted multivariable analyses (Table 3), experience of each form of stigma (anticipatory, internalized, and enacted) was associated with higher odds of poor HRQoL. Women who reported high levels of anticipatory stigma had 2.0 [95\% confidence interval (CI) 1.2, 3.6] times the odds of poor overall function and 2.1 (95\% CI 1.2, 3.7) times the odds of poor life satisfaction compared to women who reported low levels of anticipatory stigma. A similar relationship between high anticipatory stigma and poor overall function and poor life satisfaction was found among men
(Table 3). Women who reported high levels of internalized stigma had $2.2(95 \%$ CI 1.2, 4.1) times the odds of poor overall function and $2.2(95 \%$ CI 1.2, 4.0) times the odds of poor life satisfaction compared to women who reported low levels of internalized stigma. A similar relationship between high internalized stigma and both poor overall function and poor life satisfaction was found among men (Table 3). Enacted stigma was associated with significantly increased odds of poor life satisfaction among women [adjusted odds ratio [aOR] $2.0(95 \%$ CI 1.2, 3.4)]. However, the relationship between enacted stigma and poor life satisfaction did not reach statistical significance among men [aOR 2.1 (95\% CI 1.0, 4.7)]. Having experienced enacted stigma was not significantly associated with poor overall function among women or men.

\section{Discussion}

In a large sample of PLWH enrolling in HIV care in Tanzania, health-related quality of life was significantly lower among men as compared to women in both overall function and life satisfaction. Previous research examining the

Table 3 Sex-stratified bivariate and multivariable models of HIV-related stigma and health-related quality of life among adults enrolling in HIV care and not known to be eligible for ART in Tanzania

\begin{tabular}{|c|c|c|c|c|c|c|c|c|}
\hline & \multicolumn{4}{|c|}{ Poor overall function } & \multicolumn{4}{|c|}{ Poor life satisfaction } \\
\hline & \multicolumn{2}{|l|}{$\begin{array}{l}\text { Women } \\
n=503\end{array}$} & \multicolumn{2}{|l|}{$\begin{array}{l}\text { Men } \\
n=305\end{array}$} & \multicolumn{2}{|l|}{$\begin{array}{l}\text { Women } \\
\mathrm{n}=503\end{array}$} & \multicolumn{2}{|l|}{$\begin{array}{l}\text { Men } \\
\mathrm{n}=305\end{array}$} \\
\hline & $\begin{array}{l}\text { Bivariate OR } \\
(95 \% \mathrm{CI})\end{array}$ & $\begin{array}{l}\text { Multivariable } \\
\text { OR }(95 \% \mathrm{CI})^{\mathrm{a}}\end{array}$ & $\begin{array}{l}\text { Bivariate OR } \\
(95 \% \mathrm{CI})\end{array}$ & $\begin{array}{l}\text { Multivariable } \\
\text { OR }(95 \% \mathrm{CI})^{\mathrm{a}}\end{array}$ & $\begin{array}{l}\text { Bivariate OR } \\
(95 \% \mathrm{CI})\end{array}$ & $\begin{array}{l}\text { Multivariable } \\
\text { OR }(95 \% \mathrm{CI})^{\mathrm{a}}\end{array}$ & $\begin{array}{l}\text { Bivariate OR } \\
(95 \% \mathrm{CI})\end{array}$ & $\begin{array}{l}\text { Multivariable } \\
\text { OR }(95 \% \text { CI })^{\text {a }}\end{array}$ \\
\hline \multicolumn{9}{|c|}{ Enacted stigma $^{\mathrm{b}}$} \\
\hline No & 1.00 & 1.00 & 1.00 & 1.00 & 1.00 & 1.00 & 1.00 & 1.00 \\
\hline Yes & $\begin{array}{l}1.54(0.91, \\
2.62)\end{array}$ & $\begin{array}{l}1.62(0.93, \\
2.81)\end{array}$ & $\begin{array}{l}1.30(0.58, \\
2.91)\end{array}$ & $\begin{array}{l}1.23(0.52 \\
2.94)\end{array}$ & $\begin{array}{l}1.93(1.15 \\
3.22)^{*}\end{array}$ & $\begin{array}{l}2.01(1.20 \\
3.38)^{*}\end{array}$ & $\begin{array}{l}2.01(0.95 \\
4.26)\end{array}$ & $\begin{array}{l}2.13(0.96 \\
4.70)\end{array}$ \\
\hline \multicolumn{9}{|c|}{ Internalized stigma $^{c}$} \\
\hline Low & 1.00 & 1.00 & 1.00 & 1.00 & 1.00 & 1.00 & 1.00 & 1.00 \\
\hline Medium & $\begin{array}{l}0.74(0.45 \\
1.22)\end{array}$ & $\begin{array}{l}0.74(0.44 \\
1.24)\end{array}$ & $\begin{array}{l}1.05(0.60 \\
1.82)\end{array}$ & $\begin{array}{l}0.97(0.55 \\
1.73)\end{array}$ & $\begin{array}{l}0.91(0.56 \\
1.47)\end{array}$ & $\begin{array}{l}0.85(0.52 \\
1.41)\end{array}$ & $\begin{array}{l}0.99(0.56 \\
1.75)\end{array}$ & $\begin{array}{l}0.95(0.52 \\
1.71)\end{array}$ \\
\hline High & $\begin{array}{l}1.94(1.08 \\
3.49)^{*}\end{array}$ & $\begin{array}{c}2.18(1.17 \\
4.05)^{*}\end{array}$ & $\begin{array}{c}2.53(1.09 \\
5.91)^{*}\end{array}$ & $\begin{array}{c}2.58(1.06 \\
6.26)^{*}\end{array}$ & $\begin{array}{l}2.31(1.30 \\
4.12)^{*}\end{array}$ & $\begin{array}{c}2.18(1.19 \\
3.98)^{*}\end{array}$ & $\begin{array}{l}4.42(1.87 \\
10.47)^{*}\end{array}$ & $\begin{array}{l}4.94(2.02 \\
12.09)^{*}\end{array}$ \\
\hline \multicolumn{9}{|c|}{ Anticipatory stigma ${ }^{\mathrm{d}}$} \\
\hline Low & 1.00 & 1.00 & 1.00 & 1.00 & 1.00 & 1.00 & 1.00 & 1.00 \\
\hline Medium & $\begin{array}{l}1.59(0.92, \\
2.75)\end{array}$ & $\begin{array}{l}1.37(0.77 \\
2.42)\end{array}$ & $\begin{array}{l}2.56(1.39 \\
4.72)^{*}\end{array}$ & $\begin{array}{l}2.30(1.22 \\
4.37)^{*}\end{array}$ & $\begin{array}{l}1.88(1.09 \\
3.24)^{*}\end{array}$ & $\begin{array}{l}1.71(0.98, \\
2.97)\end{array}$ & $\begin{array}{l}1.88(1.01 \\
3.48)^{*}\end{array}$ & $\begin{array}{l}1.75(0.93 \\
3.32)\end{array}$ \\
\hline High & $\begin{array}{l}2.18(1.26 \\
3.77)^{*}\end{array}$ & $\begin{array}{c}2.03(1.15 \\
3.60)^{*}\end{array}$ & $\begin{array}{l}2.18(1.11 \\
4.28)^{*}\end{array}$ & $\begin{array}{l}1.90(1.04 \\
3.85)^{*}\end{array}$ & $\begin{array}{c}2.35(1.36 \\
4.07)^{*}\end{array}$ & $\begin{array}{c}2.13(1.22 \\
3.71)^{*}\end{array}$ & $\begin{array}{l}2.31(1.19 \\
4.49)^{*}\end{array}$ & $\begin{array}{l}2.13(1.06 \\
4.27)^{*}\end{array}$ \\
\hline
\end{tabular}

$* p<0.05$

${ }^{a}$ Adjusted for age, marital status, and employment

${ }^{\mathrm{b}}$ Models include enacted stigma, but not anticipatory or internalized stigma

${ }^{\mathrm{c}}$ Models include internalized stigma, but not anticipatory or enacted stigma

${ }^{\mathrm{d}}$ Models includes anticipatory stigma, but not internalized or enacted stigma 
relationship between gender and HRQoL has found gender to be consistently associated with HRQoL among PLWH. However, the directionality of this relationship has varied across studies. Similar to current findings, studies with individuals initiating ART in Ethiopia and with individuals testing HIV-positive in Kenya found that HRQoL was higher among women than men [13, 28]. However, another study with ART patients in Ethiopia found that men reported significantly higher quality of life compared to women [29]. The mechanisms through which gender influences HRQoL across settings and populations warrant further investigation.

High levels of anticipatory stigma were significantly associated with poor overall function and poor life satisfaction among both women and men. The authors were unable to identify previous research on the relationship between anticipatory HIV-related stigma and HRQoL from sub-Saharan Africa. However, among PLWH in Sweden, anticipatory stigma, measured by concerns about public attitudes, was significantly associated with poor physical HRQoL [30]. In this same study, though, concern regarding disclosure of one's HIV status, another component of anticipatory HIVrelated stigma, was not significantly associated with worse HRQoL [30]. Among PLWH in the United States, disclosure concerns were significantly associated with poor HRQoL among men and women [20]. Future research should examine to what extent the relationship between anticipatory stigma and HRQoL varies by the component of anticipatory stigma assessed (e.g., disclosure concerns, concerns about public attitudes). Factors that mediate or moderate the relationship between anticipatory stigma and HRQoL should also be examined, including whether such mediating or moderating factors differ by gender. For example, effective coping strategies or greater social support may reduce the impact of anticipatory stigma on poor HRQoL while maladaptive coping mechanisms, limited social support, or social isolation may have a synergistic effect on the relationship between anticipatory stigma and HRQoL. Greater understanding of the relationship between anticipatory stigma and HRQoL can lead to the identification of potential intervention targets to mitigate the impact of anticipatory stigma on HRQoL and improve the quality of life of PLWH. Such research is particularly needed with PLWH in sub-Saharan Africa where the prevalence and impact of HIV-related stigma remain high [31-33].

Internalized HIV-related stigma was associated with poor overall functioning and life satisfaction among both men and women. While research into the relationship between internalized HIV-related stigma and HRQoL in sub-Saharan Africa remains limited, a study of PLWH in Spain similarly found that internalized stigma was associated with worse HRQoL [34]. This study also found that the relationship between internalized stigma and HRQoL was mediated by group identity, suggesting that interventions to foster social cohesion and increase social support may be a promising strategy to mitigate the negative impact of internalized stigma on HRQoL [34]. Peer support interventions may be particularly effective at reducing the prevalence and impact of internalized HIV-related stigma, particularly as a longitudinal study with PLWH in Uganda found that internalized stigma was associated with subsequent low levels of social support [35]. However, findings related to the relationship of peer support and HIV treatment outcomes remain equivocal [36-38]. Internalized stigma has been consistently associated with poor mental health, including depressive symptoms and poor emotional well-being. Examination of the extent to which psychological distress mediates the relationship between internalized HIV-related stigma and poor HRQoL and whether this relationship differs by gender is warranted. Evidence-based mental health interventions for PLWH should consider incorporating components to reduce and manage internalized HIV-related stigma and adapted interventions should be implemented and evaluated.

In multivariable analyses, enacted stigma was not significantly associated with overall functioning or life satisfaction among men. However, enacted stigma was significantly associated with poor life satisfaction among women. Previous research into the relationship between enacted HIV-related stigma and HRQoL remains equivocal. Among PLWH in Spain, enacted stigma was significantly associated with worse HRQoL [34]. However, no significant relationship between enacted stigma and HRQoL was found when examined among PLWH in Sweden [30]. Neither study examined the relationship between enacted stigma and HRQoL separately by gender. However, research with women living with HIV in the United States found that women were particularly concerned about the negative social consequences of HIV-related stigma [22]. Similarly, research in South Africa found that the level of HIV-related stigma reported by one's community, operationalized as the percent of people in a village endorsing HIV-related stigma, was negatively associated with HIV testing among women, but not men [39]. Gender differences in the relationship between enacted stigma and HRQoL may be influenced by differences in the social positioning and socialization of women compared to men. If women place greater emphasis on preserving social relationships, women may be more negatively impacted by stigmatizing views and behaviors of community members compared to men. Additional research into the relationship between gender, enacted stigma, and HRQoL is needed.

This work has limitations worth noting. First, we assessed the relationship between HIV-related stigma and HRQoL prior to ART initiation. Future work should assess this relationship across the care continuum and examine whether there are critical points (e.g., diagnosis, disclosure) at which PLWH are particularly vulnerable to the negative effects of stigma and poor HRQoL. Additionally, given the 
cross-sectional nature of our study, temporality of the relationship between HIV-related stigma and HRQoL could not be established. It is possible that people with poor HRQoL are more sensitive to experiences of HIV-related stigma. Longitudinal research is needed to better understand pathways between HIV-related stigma and HRQoL. Further, data were collected from individuals at four HIV clinics in Tanzania. As such, results cannot be generalized to PLWH in other settings or PLWH not engaged in care. As both HIV-related stigma and HRQoL may pose barriers to engagement in care, the relationship between HIV-related stigma and HRQoL may be meaningfully different among PLWH not engaged in care. In addition, anticipatory stigma items in this study did not assess anticipatory stigma specifically in a health care setting or from health care workers. Future research should examine anticipatory stigma specifically in relation to health care settings. Finally, this analysis examined two aspects of HRQoL. Future work should examine the relationship between HIV-related stigma and other components of HRQoL.

HIV-related stigma was associated with worse HRQoL among men and women. More nuanced understanding of the longitudinal pathways between stigma and poor HRQoL can inform intervention development and adaptation. Interventions to enhance the HRQoL of PLWH should incorporate stigma reduction components. Psychosocial interventions to prevent or reduce the impact of internalized and anticipatory stigma may improve HRQoL for PLWH and should be considered across a range of HIV services. More research is needed to understand to what extent the mechanisms between these types of stigma and HRQoL differ by gender and whether gender-specific anticipatory and internalized stigma reduction intervention strategies are warranted. Antistigma interventions at the community level are also needed and may particularly benefit women living with HIV.

Acknowledgements This project was supported by a research grant from the National Institute of Mental Health (Supplement to Grant Number R01MH089831). The clinics included in this analysis received support from ICAP at Columbia University through funding from the President's Emergency Plan for AIDS Relief. The content is solely the responsibility of the authors and does not necessarily represent the official views of the funders. The funders had no role in study design, data collection and analysis, decision to publish, or preparation of the manuscript.

\section{Compliance with Ethical Standards}

Conflict of interest The authors declare they have no conflict of interest.

Ethical Approval All procedures performed in this study involving human participants were in accordance with the ethical standards of the institutional and/or national research committee and with the 1964 Helsinki declaration and its later amendments or comparable ethical standards.
Informed Consent Informed consent was obtained from all participants included in the study.

\section{References}

1. World Health Organization. Guideline on when to start antiretroviral therapy and on pre-exposure prophylaxis for HIV. Geneva: World Health Organization; 2015.

2. World Health Organization. Progress report 2016: prevent HIV, test and treat all. Geneva: World Health Organization; 2016.

3. Lundgren JD, Babiker AG, Gordin F, Emery S, Grund B, Sharma $\mathrm{S}$, et al. Initiation of antiretroviral therapy in early asymptomatic HIV infection. New Engl J Med. 2015;373(9):795-807.

4. Danel C, Moh R, Gabillard D, Badje A, Le Carrou J, Ouassa $\mathrm{T}$, et al. A trial of early antiretrovirals and isoniazid preventive therapy in Africa. New Engl J Med. 2015;373(9):808-22.

5. Cohen MS, Chen YQ, McCauley M, Gamble T, Hosseinipour MC, Kumarasamy N, et al. Prevention of HIV-1 infection with early antiretroviral therapy. New Engl J Med. 2011;365(6):493-505.

6. Nakagawa F, May M, Phillips A. Life expectancy living with HIV: recent estimates and future implications. Curr Opin Infect Dis. 2013;26(1):17-25.

7. Nsanzimana S, Remera E, Kanters S, Chan K, Forrest JI, Ford N, et al. Life expectancy among HIV-positive patients in Rwanda: a retrospective observational cohort study. Lancet Glob Health. 2015;3(3):e169-77.

8. Deeks SG, Lewin SR, Havlir DV. The end of AIDS: HIV infection as a chronic disease. Lancet. 2013;382(9903):1525-33.

9. Centers for Disease Control and Prevention. Measuring healthy days: population assessment of health-related quality of life. Atlanta, Georgia; 2000.

10. Mannheimer SB, Matts J, Telzak E, Chesney M, Child C, Wu AW, et al. Quality of life in HIV-infected individuals receiving antiretroviral therapy is related to adherence. AIDS Care. 2005;17(1):10-22.

11. de Boer-van der Kolk IM, Sprangers MA, Prins JM, Smit C, de Wolf F, Nieuwkerk PT. Health-related quality of life and survival among HIV-infected patients receiving highly active antiretroviral therapy: a study of patients in the AIDS Therapy Evaluation in the Netherlands (ATHENA) Cohort. Clin Infect Dis. 2010;50(2):255-63.

12. Pokhrel KN, Sharma VD, Shibanuma A, Pokhrel KG, Mlunde LB, Jimba M. Predicting health-related quality of life in people living with HIV in Nepal: mental health disorders and substance use determinants. AIDS Care. 2017;29(9):1137-43.

13. Vo QT, Hoffman S, Nash D, El-Sadr WM, Tymejczyk OA, Gadisa $\mathrm{T}$, et al. Gender differences and psychosocial factors associated with quality of life among ART initiators in Oromia, Ethiopia. AIDS Behav. 2016;20(8):1682-91.

14. Rueda S, Mitra S, Chen S, Gogolishvili D, Globerman J, Chambers L, et al. Examining the associations between HIV-related stigma and health outcomes in people living with HIV/AIDS: a series of meta-analyses. BMJ Open. 2016;6(7):e011453.

15. Ekstrand ML, Heylen E, Mazur A, Steward WT, Carpenter C, Yadav K, et al. The role of HIV stigma in ART adherence and quality of life among rural women living with HIV in India. AIDS Behav. 2018;22(12):3859-68.

16. Shrestha S, Shibanuma A, Poudel KC, Nanishi K, Koyama Abe M, Shakya SK, et al. Perceived social support, coping, and stigma on the quality of life of people living with HIV in Nepal: a moderated mediation analysis. AIDS Care. 2019;31(4):413-20. 
17. Earnshaw VA, Chaudoir SR. From conceptualizing to measuring HIV stigma: a review of HIV stigma mechanism measures. AIDS Behav. 2009;13(6):1160-77.

18. Sherwood J, Sharp A, Cooper B, Roose-Snyder B, Blumenthal S. HIV/AIDS National Strategic Plans of Sub-Saharan African countries: an analysis for gender equality and sex-disaggregated HIV targets. Health Policy Plan. 2017;32(10):1361-7.

19. Remien RH, Chowdhury J, Mokhbat JE, Soliman C, Adawy ME, El-Sadr W. Gender and care: access to HIV testing, care, and treatment. J Acquir Immune Defic Syndr. 2009;51(Suppl 3):S106-10.

20. Fekete EM, Williams SL, Skinta MD, Bogusch LM. Gender differences in disclosure concerns and HIV-related quality of life. AIDS Care. 2016;28(4):450-4.

21. Li L, Lin C, Ji G. Gendered aspects of perceived and internalized HIV-related stigma in China. Women Health. 2017;57(9):1031-43.

22. Sandelowski M, Lambe C, Barroso J. Stigma in HIV-positive women. J Nurs Scholarsh. 2004;36(2):122-8.

23. Holmes WC, Shea JA. Performance of a new, HIV/AIDS-targeted quality of life (HAT-QoL) instrument in asymptomatic seropositive individuals. Qual Life Res. 1997;6(6):561-71.

24. Holmes WC, Bilker WB, Wang H, Chapman J, Gross R. HIV/ AIDS-specific quality of life and adherence to antiretroviral therapy over time. J Acquir Immune Defic Syndr. 2007;46(3):323-7.

25. Taylor TN, Dolezal C, Tross S, Holmes WC. Reliability and validity of two HIV/AIDS-specific quality of life instruments adapted for use in HIV-positive Zimbabweans. AIDS Care. 2009;21(5):598-607.

26. Phaladze NA, Human S, Dlamini SB, Hulela EB, Hadebe IM, Sukati NA, et al. Quality of life and the concept of "living well" with HIV/AIDS in sub-Saharan Africa. J Nurs Scholarsh. 2005;37(2):120-6.

27. Holzemer WL, Uys LR, Chirwa ML, Greeff M, Makoae LN, Kohi TW, et al. Validation of the HIV/AIDS Stigma InstrumentPLWA (HASI-P). AIDS Care. 2007;19(8):1002-12.

28. van der Kop ML, Muhula S, Patel A, Thabane L, Awiti P, Kyomuhangi $\mathrm{L}$, et al. Gender differences in health-related quality of life at the time of a positive HIV test-a cross-sectional study in a resource-poor, high prevalence setting in Nairobi, Kenya. AIDS Care. 2018;30(4):493-9.

29. Tesfay A, Gebremariam A, Gerbaba M, Abrha H. Gender differences in health related quality of life among people living with HIV on highly active antiretroviral therapy in Mekelle Town, Northern Ethiopia. Biomed Res Int. 2015;2015:516369.

30. Reinius M, Wiklander M, Wettergren L, Svedhem V, Eriksson LE. The relationship between stigma and health-related quality of life in people living with HIV who have full access to antiretroviral treatment: an assessment of Earnshaw and Chaudoir's HIV stigma framework using empirical data. AIDS Behav. 2018;22(12):3795-806.

31. Endeshaw M, Walson J, Rawlins S, Dessie A, Alemu S, Andrews $\mathrm{N}$, et al. Stigma in Ethiopia: association with depressive symptoms in people with HIV. AIDS Care. 2014;26(8):935-9.

32. Feyissa GT, Abebe L, Girma E, Woldie M. Stigma and discrimination against people living with HIV by healthcare providers, Southwest Ethiopia. BMC Public Health. 2012;12:522.

33. Odimegwu CO, Akinyemi JO, Alabi OO. HIV-stigma in Nigeria: review of research studies, policies, and programmes. AIDS Res Treat. 2017;2017:5812650.

34. Fuster-Ruizdeapodaca MJ, Molero F, Holgado FP, Mayordomo $S$. Enacted and internalized stigma and quality of life among people with HIV: the role of group identity. Qual Life Res. 2014;23(7):1967-75.

35. Tsai AC, Bangsberg DR, Emenyonu N, Senkungu JK, Martin JN, Weiser SD. The social context of food insecurity among persons living with HIV/AIDS in rural Uganda. Soc Sci Med. 2011;73(12):1717-24.

36. Elul B, Basinga P, Nuwagaba-Biribonwoha H, Saito S, Horowitz D, Nash D, et al. High levels of adherence and viral suppression in a nationally representative sample of HIV-infected adults on antiretroviral therapy for 6, 12 and 18 months in Rwanda. PLoS ONE. 2013;8(1):e53586

37. Chang LW, Kagaayi J, Nakigozi G, Ssempijja V, Packer AH, Serwadda D, et al. Effect of peer health workers on AIDS care in Rakai, Uganda: a cluster-randomized trial. PLoS ONE. 2010;5(6):e10923.

38. Pearson CR, Micek MA, Simoni JM, Hoff PD, Matediana E, Martin DP, et al. Randomized control trial of peer-delivered, modified directly observed therapy for HAART in Mozambique. J Acquir Immune Defic Syndr. 2007;46(2):238-44.

39. Treves-Kagan S, El Ayadi AM, Pettifor A, MacPhail C, Twine R, Maman S, et al. Gender, HIV testing and stigma: the association of HIV testing behaviors and community-level and individuallevel stigma in rural South Africa differ for men and women. AIDS Behav. 2017;21(9):2579-88.

Publisher's Note Springer Nature remains neutral with regard to jurisdictional claims in published maps and institutional affiliations. 